\title{
Phase Modulation Technique for Rotation Rate Sensing Based on High Quality Optical Resonator
}

\author{
Xu Pengfei $i^{1, a}$, Xiao Jun ${ }^{1, b}$, Peng $\mathrm{Qi}^{1, \mathrm{c}}$, Huang Linyi ${ }^{1, \mathrm{~d}}$ and Hu jianyao ${ }^{1, \mathrm{e}}$ \\ ${ }^{1}$ The Fifth Electronics Research Institute of Ministry of Industry and Information Technology \\ xupengfei@ceprei.biz, ${ }^{b} x i a o j u n @ c e p r e i . b i z,{ }^{c}$ pengqi@ceprei.biz,

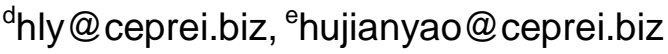

Keywords: phase modulation, rotation sensing, optical resonator.

Abstract. Three kinds of phase modulation methods adopted in the rotation rate sensor based on microsphere resonator are investigated. The performance of these modulation methods were compared and analyzed. As a result, sinusoidal wave modulation was employed and the sidebands demodulation has been realized in the sensing system which provide a valuable reference for signal processing of rotation rate sensors.

\section{Introduction}

The optical microsphere resonators made by fused silica have attracted much interests for their high quality factor and small mode volume ${ }^{[1]}$. They are widely used in the different kinds of sensor applications, such as micro-optical force sensors ${ }^{[2]}$, temperature sensors ${ }^{[3]}$ and biochemical sensors ${ }^{[4]}$. Additionally, the micro-scale optical resonator can be applied to realize rotation sensing, which is critical important in the field of inertial navigation and guidance, according to the Sagnac effect. The rotation rate can be obtained by detecting the resonant frequency difference between counterpropagating light in the microsphere resonator. Due to the weak frequency shift introducing by Sagnac effect, phase modulation technique must be employed to replace the direct measurement method. By modulating the light injected into the microsphere resonator, demodulation signal, which reveals the relationship of rotation rate and sensor output, can be acquired by demodulation function module.

However, the adoption of different phase modulation methods will have kinds of influence to the signal. Here we compared there different phase modulation methods which are sawtooth wave, triangular wave and sinusoidal wave phase modulation. The high SNR modulated resonant signals and corresponding demodulation signals were achieved by using sinusoidal wave modulation which provide a reference for the choice of modulation method. Furthermore, the PDH (Pound-Drever-Hall, $\mathrm{PDH})$ technique was firstly applied and realized in the microsphere resonator rotation rate sensing system.

\section{Theory}
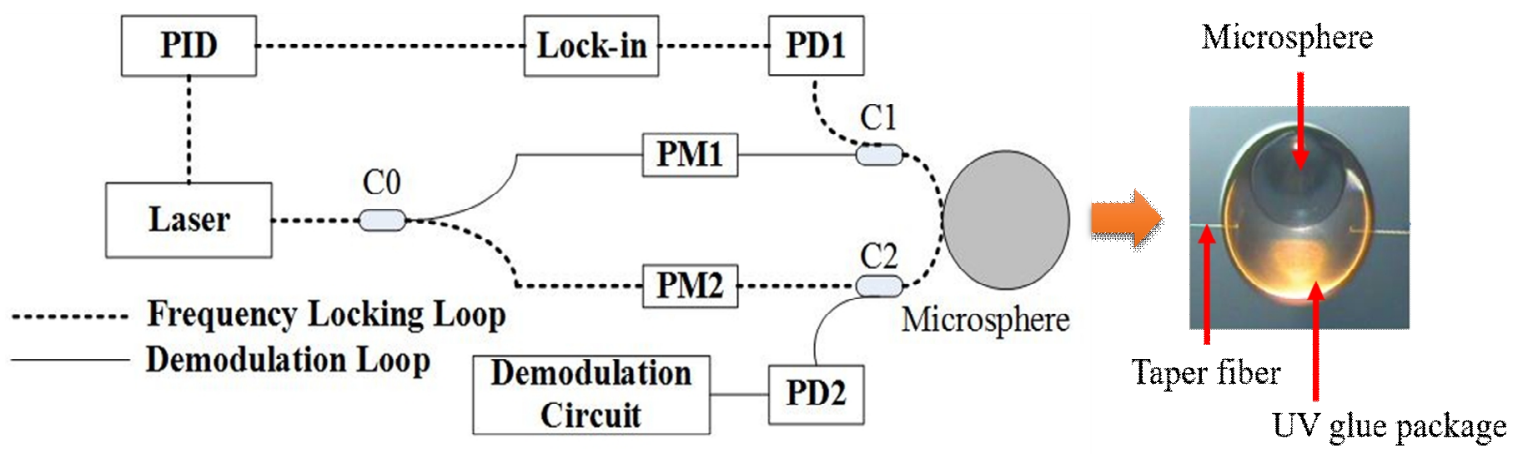

Fig. 1 Schematic diagram of rotation rate sensing system based on microsphere resonator 
The rotation rate sensing system based on the optical microsphere resonator include two parts, frequency locking loop and demodulation loop, as shown in Fig. 1. The frequency locking loop is used to fix the laser frequency at the resonant frequency, along the clockwise direction, of microsphere resonator which provide a stable frequency reference for detection of resonant frequency difference between counterpropagating light. The demodulation loop will output the demodulation signal which indicates the linear relationship between rotation rate and sensor signal.

The microsphere resonator employed in the system was coupled with taper fiber and packaged by low refractive index UV glue $\left(\mathrm{n}_{\mathrm{UV}}=1.37\right)$ to improve the robustness of fiber-microsphere coupling device and get rid of the influence of air flow and vibration noises from external environment ${ }^{[5]}$. The light field enter the microsphere can be written as:

$$
E_{\text {Laser }}=E_{0} e^{i\left(w t+\varphi_{0}\right)} .
$$

Where $E_{0}$ is the amplitude of the laser, $\omega$ is the center frequency of laser, $\varphi_{0}$ is the initial phase of light. The light field output from resonator can be derived as:

$$
\begin{aligned}
& E_{\text {out }}=E_{0} e^{i\left(w t+\varphi_{0}\right)}\left[U-R \sum_{n=1}^{\infty} Q^{n-1} e^{-i(w n \pi)}\right], \\
& U=\left(1-k_{C}\right)^{1 / 2}\left(1-\alpha_{C}\right)^{1 / 2} \\
& R=k_{C}\left(1-\alpha_{C}\right)\left(1-\alpha_{L}\right)^{1 / 2} \\
& Q=\left(1-k_{C}\right)^{1 / 2}\left(1-\alpha_{C}\right)^{1 / 2}\left(1-\alpha_{L}\right)^{1 / 2} .
\end{aligned}
$$

Where $\tau$ is time that ligth propagate one circle in the resonator, $k_{C}$ is the coupling coefficient, $\alpha_{C}$ is the loss coefficient of the coupler, $\alpha_{L}$ is the loss coefficient of the resonator, $\mathrm{n}$ is the times that light pass the coupler. $U$ is the coefficient for light directly coupling out of the resonator, $\mathrm{R}$ is the cross coupling coefficient and Q is the one-circle coefficeient. Thus, the transfer function of microsphere can be obtained as:

$$
H(w)=\frac{E_{\text {out }}}{E_{\text {Laser }}}=U-R \frac{1-Q^{n} e^{-i(w n / F S R)}}{1-Q e^{-i(w / F S R)}} e^{-i(w / F S R)} .
$$

Where FSR (Free Spectrum Range, FSR) represents the free spectrum range of resonant spectrum for microsphere resonator.

\section{Experiments}

In the experiments, the narrow linewidth laser (NKT Photonics, linewidth $<1 \mathrm{kHz}$ ) was employed with center wavelength at $1550 \mathrm{~nm}$. The two phase modulators (Thorlabs LN65S) with modulation frequency about $10 \mathrm{GHz}$ and lock-in amplifier (Stanford SR844) with frequency range $20 \mathrm{kHz} 200$ $\mathrm{MHz}$ were adopted. By applying different types of modulation signals to the modulator PM2, the investigation of the performance for phase modulation can be carried out.

The resonant spectrum shown in Fig. 2a was modulated by the sawtooth wave with peak-peak amplitude $4 \mathrm{~V}$ and frequency $100 \mathrm{kHz}$. For the sharp reset of amplitude in sawtooth wave, obvious restoration error signal appears along the modulated resonant spectrum which make the SNR dramatically decrease. By changing the frequency and amplitude of sawtooth, the SNR of modulated resonant spectrum under different conditions were investigated. As depicted in Fig. 2b, the SNR reduced as the amplitude of sawtooth wave increase regardless of frequency. On the other hand, for modulation frequency, $100 \mathrm{kHz}, 900 \mathrm{kHz}$ and $5 \mathrm{MHz}$, the SNR shows none improvement with the change of sawtooth wave amplitude. The signal with worse SNR will lead to the signal process module complex and cause serious error in the sensing system. 
(a)

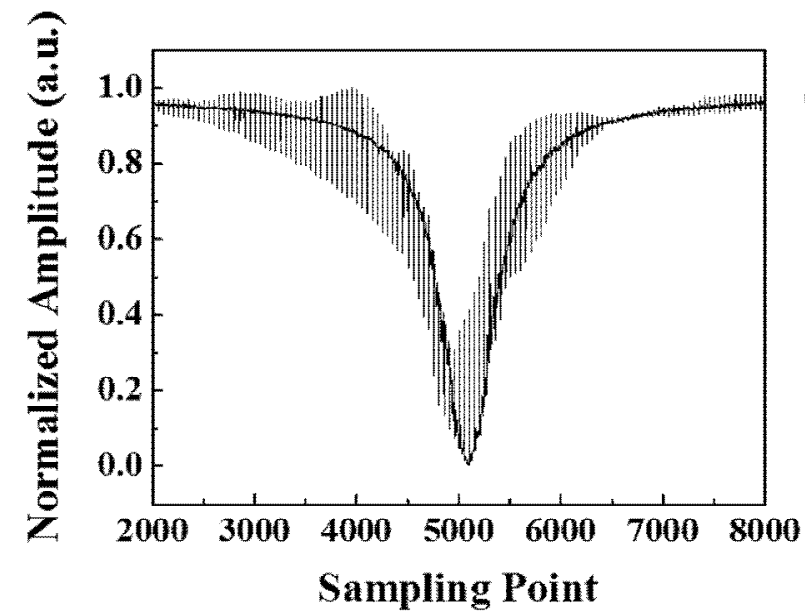

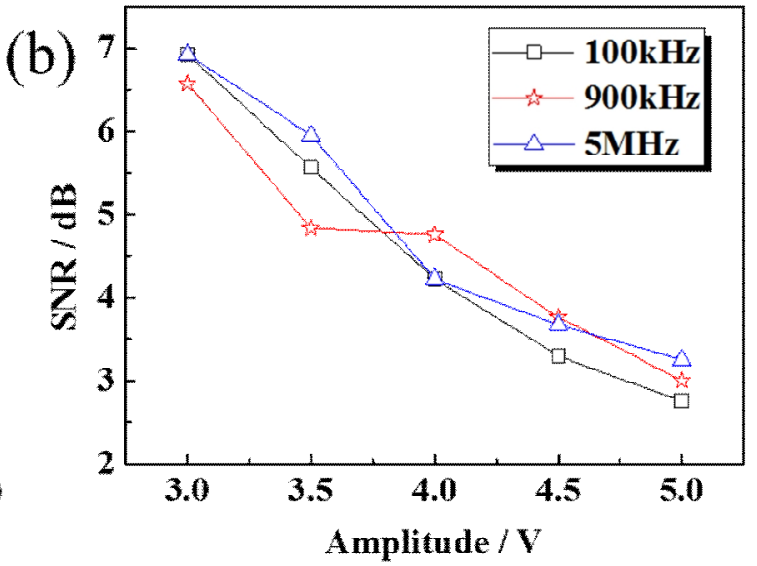

Fig. 2 (a) resonant spectrum modulated by sawtooth wave with frequency $100 \mathrm{kHz}$. (b) SNR of resonant spectra modulated by different frequency sawtooth waves with different amplitude.

While the resonant signal is modulated by the triangular wave with peak-peak amplitude $3 \mathrm{~V}$ and frequency $1 \mathrm{MHz}$, the restoration errors disappear as shown in Fig. 3a. For triangular wave, the amplitude is changing slowly which avoid the influence of sharp reset. However, some kinds of noised still exist in the modulated signal. As the section marked in the Fig. 3a is enlarged, shown in Fig. $3 b$, square waves emerge with the same frequency to the triangular wave. According to the resonator modulation theory, the frequency of modulated light will have a small shift. When the light come out of the resonator, light intensity will be different as the frequency changes. Thus, the light frequency shift finally induced the change of amplitude which is obtained by photodetector. However, the distortions in the square wave, which are caused by signal overshoot or undershoot occur, will lead to the accuracy decrease. Oversampling and algorithm have to be taken to solve the problem.
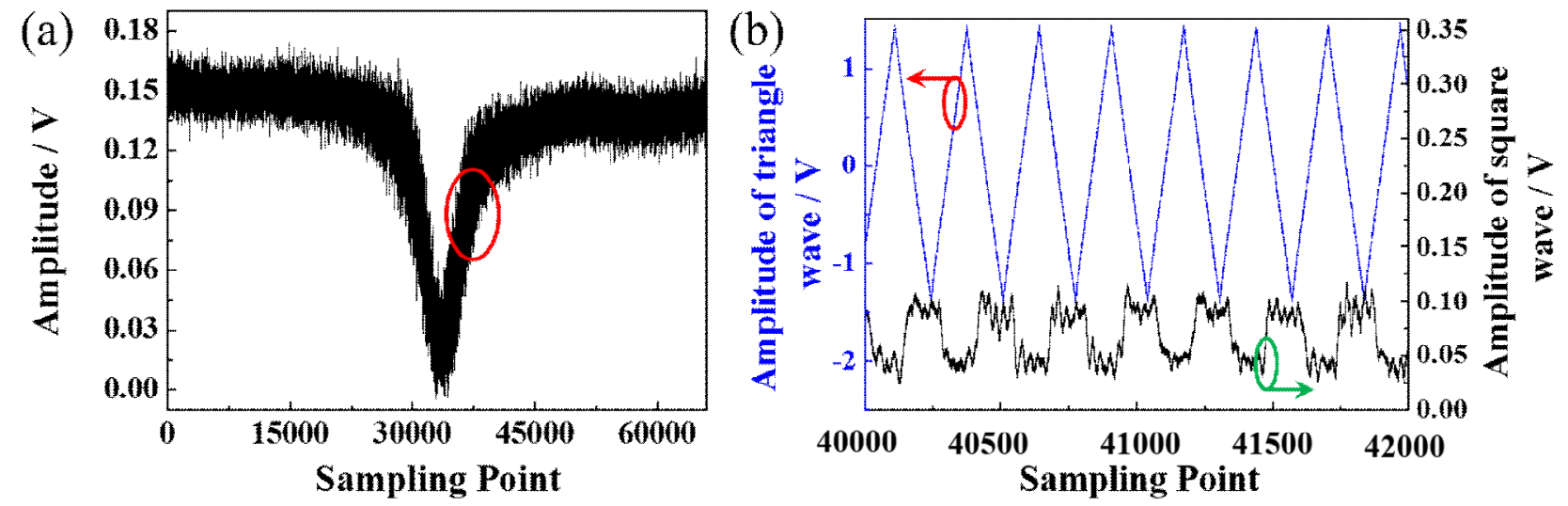

Fig.3 (a) resonant spectrum modulated by triangular wave with frequency $1 \mathrm{MHz}$. (b) enlarged view of the curve marked in Fig.3a.

Furthermore, the sinusoidal wave with peak-peak amplitude $4 \mathrm{~V}$ and frequency $900 \mathrm{kHz}$ was adopted. It's obviously that the SNR of the modulated signal is improved, compared to the others mentioned above, as shown in Fig. 4a. To investigate the performance of different modulation methods, these three kinds of modulation signals with $900 \mathrm{kHz}$ and different amplitude were applied to the phase modulator respectively. As revealed by Fig. 4b, the SNR of resonant spectrum modulated by sinusoidal wave is higher, about $20 \mathrm{~dB}$ in average, than sawtooth wave and triangular wave. Considering the flexibility and high SNR in modulation process, sinusoidal wave provide a better choice to realized phase modulation. 

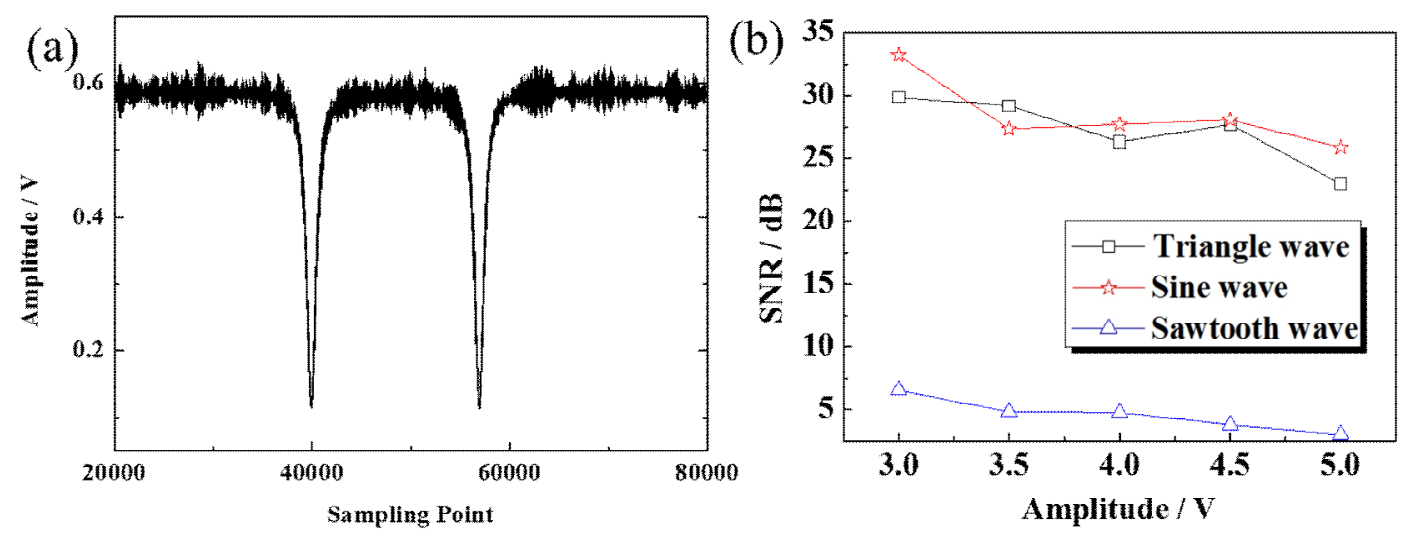

Fig.4 (a) resonant spectrum modulated by sinusoidal wave with frequency $900 \mathrm{kHz}$. (b) SNR comparison for resonant spectrum modulated by different amplitude waveform with $900 \mathrm{kHz}$.

To further verify the feasibility of sinusoidal wave modulation method, the demodulation signal was obtained, as shown in Fig. 5a, and original signal without any post process appears smooth which indicates high SNR. When the modulation frequency increased to $15 \mathrm{MHz}$ with amplitude $4 \mathrm{~V}$, sidebands of the resonant dip emerge and the corresponding demodulation signal changes as well. The high frequency modulation induced sidebands phenomenon can be used as PDH technique applying to the laser frequency lock. These high performance of demodulation signals revealed that sinusoidal wave modulation method own significant advantages.
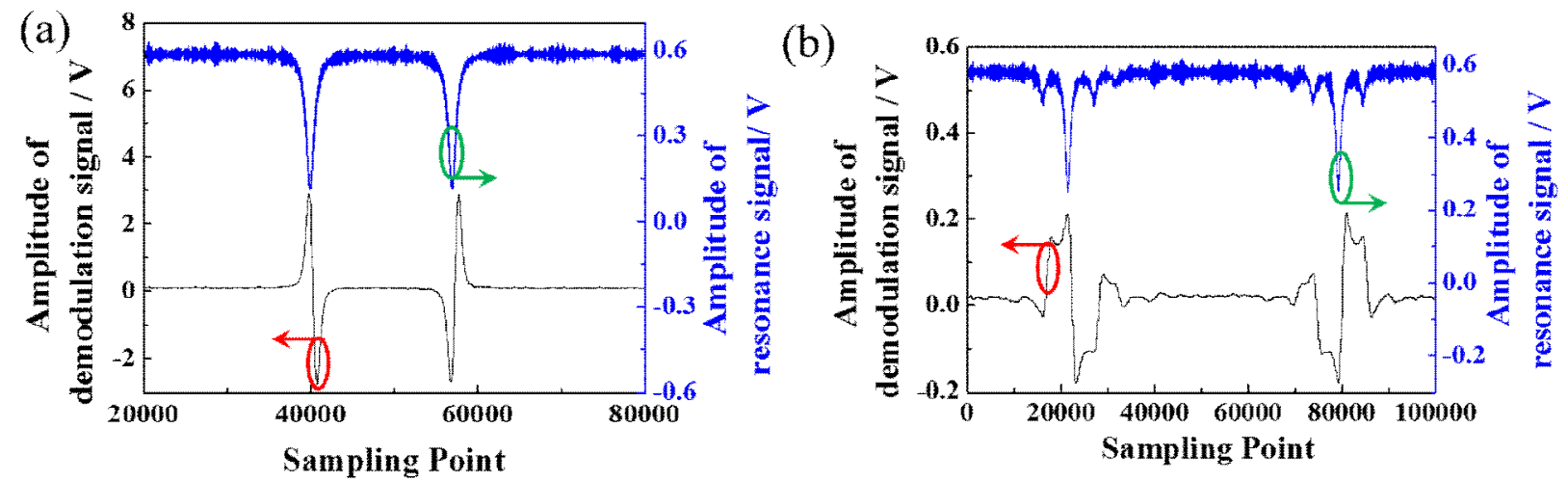

Fig.5 demodulation signal for modulation frequency at (a) $900 \mathrm{kHz}$ and (b) $15 \mathrm{MHz}$.

\section{Conclusion}

In this paper, we have investigated three kinds of phase modulation methods, sawtooth wave modulation, triangular wave modulation and sinusoidal wave modulation, adopted in the rotation rate sensor based on microsphere resonator. The performance of these modulation methods were compared and analyzed. As the better choice, sinusoidal wave modulation was employed and the sidebands demodulation has been realized in the sensing system which provide a valuable reference for signal processing of rotation rate sensors.

\section{References}

[1] M. Cai, O. Painter and K. J. Vahala: Physical Review Letters, 85(1) (2000), p. 74

[2] T. Loppolo, M. Kozhevnikov and V. Stepaniuk: Applied Optics, 47(16) (2008) p.3009

[3] D. V. Strekalov, R. J. Thompson and L. M. Baumgartel: Optics Express, 19(15) (2011) p.14495

[4] E. Chow, A. Grot and L. W. Mirkarimi: Optics Letters, 29(10) (2004) p.1093

[5] Y. Z. Yan, C. L. Zou and S. B. Yan: Optics Express. 19(7) (2011) p.5753 